\title{
Das Potenzial Positiver Psychologie in der Arbeitswelt
}

\author{
Carmen Elisabeth Lobbe • Kathrin Reinke
}

Eingegangen: 8. Oktober 2021 / Angenommen: 21. Oktober 2021 / Online publiziert: 21. Dezember 2021 (C) Der/die Autor(en) 2021

Zusammenfassung Das Wohlbefinden von Mitarbeitenden ist ein entscheidender Einflussfaktor für diverse organisationale Erfolgsgrößen wie Arbeitsleistung oder -zufriedenheit. Dieser Artikel beschäftigt sich mit der Frage, ob und durch welche Wirkmechanismen Interventionen der Positiven Psychologie das Wohlbefinden in Arbeitnehmerstichproben erhöhen und organisationale Ergebnisgrößen beeinflussen können. Die Studienergebnisse unter Einbezug bisheriger Forschung legen nahe, dass Positive Interventionen bei langfristiger Anwendung das Potenzial haben, Wohlbefinden und persönliche Ressourcen von Beschäftigten zu steigern. Implikationen für die Praxis werden diskutiert.

Schlüsselwörter Positive Psychologie $\cdot$ Positive Interventionen · Broaden and Build Theory

Carmen Elisabeth Lobbe $(\square)$

Fachgebiet Wirtschaftspsychologie, Universität Kassel, Pfannkuchenstraße 1, 34109 Kassel, Deutschland

E-Mail: lobbe.carmen@gmail.com

Dr. Kathrin Reinke

Institut für Psychologie, Technische Universität Darmstadt, Alexanderstraße 10, 64289 Darmstadt, Deutschland

E-Mail: kathrin.reinke@tu-darmstadt.de 


\title{
The potential of positive psychology in the world of work
}

\begin{abstract}
In the work context, employee well-being is a decisive factor influencing various organizational outcome variables, such as work performance and job satisfaction. The present article deals with the question if and through which mechanisms positive psychology interventions impact well-being in employee samples and influence organizational outcome variables. Results of the study integrated with previous research suggest that with a long-term use, positive psychology interventions have the potential to foster well-being and personal resources of employees. Implications for practice are discussed.
\end{abstract}

Keywords Positive Psychology $\cdot$ Positive Interventions $\cdot$ Broaden and Build Theory

\section{Wohlbefinden als Erfolgsgarant? - Bestandsaufnahme und Forschungsansatz}

Menschen mit hohem Wohlbefinden sind erfolgreicher in der Arbeitswelt als ihre weniger glückliche Kollegenschaft. Diese These lässt sich aus dem aktuellen Forschungsstand zu Wohlbefinden von Mitarbeitenden schließen: Metaanalysen unterstreichen dessen positive Zusammenhänge mit der Gesundheit, der Qualität zwischenmenschlicher Beziehungen und der Arbeitsleistung (Kaplan et al. 2009; Lyubomirsky et al. 2005a). Für Unternehmen ist ein hohes Wohlbefinden der Beschäftigten auch mit einem ökonomischen Nutzen verbunden, da es mit einer geringeren Fluktuationsabsicht und höherer Arbeitsleistung einhergeht (Lyubomirsky et al. 2005a; Wright und Bonett 2007). Andererseits kann Arbeitsstress zu psychischen und physischen Erkrankungen, wie z.B. Depressionen, und damit zu höheren Krankenversicherungskosten und Produktionsverlusten führen (Bono et al. 2013). Es lohnt sich also aus mehreren Gründen für Unternehmen, in das Wohlbefinden ihrer Mitarbeitenden zu investieren (Cropanzano und Wright 2001).

Der Ansatz, Wohlbefinden zu fördern, um auch positive Effekte auf organisationalen Erfolg auszuüben, lässt sich im Rahmen der betriebswirtschaftlich orientierten Forschungsrichtung Positive Organizational Scholarship (POS) einordnen (Kaiser et al. 2007). Diese untersucht Umstände und positive Phänomene, die zum organisationalen Erfolg beitragen. Zurückführen lässt sich diese Forschungsrichtung auf die Positive Psychologie, die mehr und mehr an Bedeutung gewinnt - für Individuen wie auch für Unternehmen, Trainer, Coaches und Therapeuten (Seligman et al. 2006; Tomoff 2017).

Die Positive Psychologie beschäftigt sich primär mit der Entstehung, Förderung und Kultivierung positiver Emotionen, da positive Emotionen als Schlüsselfaktor für die Steigerung des Wohlbefindens gelten (Tomoff 2017). Dabei werden negative Emotionen nicht negiert, sondern als akzeptierter Bestandteil des Menschen im Zusammenspiel mit positiven Emotionen untersucht. In diesem Rahmen werden Interventionen entwickelt, die befähigende Voraussetzungen für das Leben und die persönliche Entwicklung adressieren (Seligman 2010). Genau diese auf die Förde- 
rung positiver Elemente fokussierte Sichtweise revolutionierte die sonst eher defizitorientierten Ansätze der Stressmanagementforschung, die primär auf negative Faktoren der Arbeit einging und fragte, wie diese vermieden werden können (Cropanzano und Wright 2001). Neuere Befunde zeigen, dass es für die Förderung des Wohlbefindens unzureichend ist, ,nur“ das Auftreten negativer Ereignisse zu reduzieren (Bono et al. 2013). Stattdessen seien positive Ereignisse und Emotionen ganz entscheidend für die Steigerung von Wohlbefinden und damit verbundenen positiven Ergebnisgrößen, wie z. B. der Arbeitsleistung.

Forschungserkenntnisse der Positiven Psychologie konnten zeigen, dass durch gezielte Interventionen (auch: „Positive Interventionen“) das Wohlbefinden erhöht werden kann (z.B. Sin und Lyubomirsky 2009). Bisher ist jedoch kaum erforscht, wie sich diese Maßnahmen auf organisationale Ergebnisgrößen auswirken können. Aufgrund dieser Feststellung beleuchtet der vorliegende Artikel, ob und wie Positive Interventionen kritische Arbeitsgrößen wie Arbeitszufriedenheit und organisationales Commitment beeinflussen - und damit zum organisationalen Erfolg beitragen können. Der Beitrag integriert die Ergebnisse einer placebo-kontrollierten Interventionsstudie in die aktuelle Forschung und trägt dazu bei, den Nutzen Positiver Interventionen für die Arbeitswelt zu elaborieren und ihre Wirkungsmechanismen zu beleuchten. Darüber hinaus werden Unternehmen Entscheidungshilfen für oder gegen die Einbindung solcher Interventionen gegeben und Ansatzpunkte aufgezeigt, die den Erfolg Positiver Interventionen begünstigen können.

\section{Zur Effektivität Positiver Interventionen}

Verschiedene Ansätze erklären die Bedeutsamkeit von hohem Wohlbefinden. Nach der Happy-Productive Worker Thesis verfügen glückliche Mitarbeitende über mehr wertvolle Ressourcen, weshalb sie Chancen in ihrem Umfeld eher wahrnehmen, mehr aus sich herauskommen, hilfsbereiter, optimistischer und selbstbewusster sind (Cropanzano und Wright 2001). Die gute Nachricht dabei ist: Laut Studien sind rund $40 \%$ der Varianz von Wohlbefinden auf absichtliche kognitive, motivationale und verhaltensbezogene Aktivitäten zurückzuführen. Freiwillige Anstrengungen und die Änderungen dieser absichtlichen Aktivitäten sind folglich vielversprechende Möglichkeiten, um das Wohlbefinden zu steigern (Lyubomirsky et al. 2005b). Diese Erkenntnisse deuten auf das Potenzial von Interventionen für die Förderung des Wohlbefindens hin (z. B. Metaanalysen von Chakhssi et al. 2018; Sin und Lyubomirsky 2009).

Positive Interventionen sind Praktiken oder bewusste Handlungen, die positive Emotionen, Kognitionen und Verhaltensweisen fördern und so das Wohlbefinden von Menschen erhöhen können (Mitchell et al. 2010; Sin und Lyubomirsky 2009). Sie beinhalten typischerweise Aktivitäten, die Personen selbstständig durchführen können (Tetrick und Winslow 2015), und sind als individuelle, verhaltensbezogene Maßnahmen einzuordnen. Die aktuelle empirische Kenntnislage weist auf die Wirksamkeit von Positiven Interventionen hin: So zeigte eine Metaanalyse mit 51 Studien zu Interventionen aus der Positiven Psychologie, dass durch ihren Einsatz sowohl das Wohlbefinden wie z. B. positiver Affekt und Lebenszufriedenheit verbessert (Mittel 
der Effektstärke $r=0,29)$ als auch depressive Symptome verringert werden können (Mittel der Effektstärke $r=0,31$; Sin und Lyubomirsky 2009). Gander et al. (2016) zeigten ebenfalls signifikant positive Effekte von Positiven Interventionen auf das wahrgenommene Glück sowie negative Effekte auf depressive Symptome, die bis zu sechs Monate anhielten. Eine Metaanalyse mit ausschließlich klinischen Stichproben von Chakhssi et al. (2018) ergab, dass durch Positive Interventionen sowohl das Wohlbefinden verbessert als auch Depression und Angst von Patienten mit somatischen oder psychiatrischen Störungen vermindert werden kann. Darüber hinaus kann der Einsatz Positiver Interventionen mit einer Reduktion von Abwesenheitstagen von Beschäftigten aufgrund von Krankheit einhergehen (Kaplan et al. 2014). Es gibt jedoch auch kritischere Stimmen: Eine Metaanalyse von White et al. (2019) zeigte im Vergleich zu vorherigen Studien zwar signifikante, jedoch geringere Effekte Positiver Interventionen auf das Wohlbefinden (Mittel der Effektstärke $r=0,10$ ), während die Effekte auf depressive Symptome eine hohe Variabilität aufwiesen und nicht signifikant waren. Insgesamt lässt sich aus dem aktuellen Forschungsstand schließen, dass Positive Interventionen zwar eher geringe bis moderate, jedoch konsistent positive Effekte auf das Wohlbefinden haben.

\subsection{Die Three Good Things Intervention}

Die Positive Intervention „Three Good Things“ (TGT-Intervention) beinhaltet, jeden Abend drei Dinge aufzuschreiben, die an diesem Tag gut verlaufen sind, und zu reflektieren, warum diese gut verlaufen sind (Seligman et al. 2005). Weshalb die TGT-Intervention Wohlbefinden verbessern kann, wird in der Forschung bereits in Ansätzen erklärt: Laut Bono et al. (2013) werden durch die TGT-Intervention internal kognitive Prozesse angestoßen, die Menschen dazu anregen, über positive Ereignisse nachzudenken. Insbesondere positive Dinge zu reflektieren, widerspricht zunächst der natürlichen menschlichen Tendenz zur verstärkten Auseinandersetzung mit negativen Ereignissen (Seligman et al. 2006). Durch die TGT-Intervention wird diese Tendenz aktiv unterbrochen, und neue Denkmuster werden angestoßen, was laut Bono et al. (2013) zu der vermehrten Erfahrung von positiven Emotionen führe. Diese wiederum können über verschiedene Wege, wie z.B. der Verringerung von Stresssymptomen (Siu et al. 2015), das Wohlbefinden langfristig erhöhen.

Mehrere Studien zeigen, dass die TGT-Intervention zu einer Steigerung von Glück und einer Reduzierung von depressiven Symptomen führen kann (z. B. Mongrain und Anselmo-Matthews 2012; Seligman et al. 2005; Wellenzohn et al. 2016). In einer Tagebuchstudie von Bono et al. (2013) zeigte sich außerdem, dass die Auswirkung von Konflikten zwischen dem Arbeits- und dem Privatleben auf die Stimmung am Abend durch die TGT-Intervention gemindert werden kann.

\subsection{Positive Interventionen im Arbeitskontext}

Während Positive Interventionen hinsichtlich ihrer Wirkung auf das Wohlbefinden und depressive Symptome bereits oftmals untersucht wurden, ist es um andere Kontexte und Ergebnisgrößen eher schwach bestellt. Es bleibt die Frage offen, inwieweit sich Positive Interventionen auch auf erfolgskritische organisationale Ergebnisgrö- 
Ben wie Arbeitsleistung, Arbeitszufriedenheit oder Commitment auswirken (Meyers et al. 2013). Der Fokus auf Arbeitnehmerstichproben scheint ein erster wichtiger Ansatzpunkt zu sein, um Effekte im Arbeitskontext zu evaluieren und die bisherige Forschung, die primär auf unlimitierten Stichproben einschließlich Kindern sowie depressiver Probanden basiert, zu erweitern (ebd.).

Allerdings sind die Wirkungsprozesse, die die Effektivität von Positiven Interventionen erklären können, wenig erforscht (Tetrick und Winslow 2015). Die Broaden and Build Theory (BBT; Fredrickson 1998) legt nahe, dass persönliche Ressourcen wie das Psychologische Kapital eine wichtige mediierende Rolle spielen. Durch die Berücksichtigung solcher Mediatoren bei der Untersuchung von Interventionen können theoretische Wirkmechanismen beleuchtet und wertvolle Entscheidungshilfen für die Praxis identifiziert werden, z. B. bezüglich der Integration Positiver Interventionen in bestehende Stress-Management-Programme (Tetrick und Winslow 2015). Die vorliegende Studie untersucht daher die Effektivität einer Positiven Intervention, der TGT-Intervention, für eine Arbeitnehmerstichprobe sowie für arbeitsbezogene Ergebnisgrößen und bezieht dabei persönliche Ressourcen als potenziellen Mediator mit ein.

\section{Hypothesen}

\subsection{Positive Interventionen und Wohlbefinden}

Mit Hilfe der BBT werden im Folgenden die Wirkmechanismen der TGT-Intervention erklärt. Die BBT gilt als ein Grundlagenmodell zur Untersuchung Positiver Interventionen, da eben diese die Steigerung positiver Emotionen zum Ziel haben (vgl. Gander et al. 2013). Nach der BBT veranlassen positive Emotionen Menschen dazu, altbewährte automatische Verhaltensmuster zu verwerfen und neue, kreative Denk- und Handlungswege zu gehen (Fredrickson 1998). Dieses erweiterte Denkund Handlungsrepertoire geht mit neuartigen Erfahrungen einher, die über die Zeit hinweg neue Ressourcen bilden, wie z.B. eine erhöhte Selbstwirksamkeit (Ouweneel et al. 2013). Über das Wachstum der Ressourcen wiederum können Individuen bestimmte Anforderungen besser erfüllen und Probleme in stressigen Situationen eher lösen (Hobfoll 2002). Nach Fredrickson (2001) führt die durch positive Emotionen erweiterte Aufmerksamkeit und Kognition außerdem zu einer erfolgreicheren Bewältigung von Widrigkeiten, die das Individuum wiederum positive Emotionen erfahren lässt. Dieses reziproke Beziehungsgeflecht verdeutlicht einen Kreislauf, der auf Dauer die psychische Belastbarkeit und das Wohlbefinden verbessert.

Auf Grundlage der zuvor erläuterten Forschungserkenntnisse zum Potenzial Positiver Interventionen, positive Emotionen zu fördern, und der BBT wird Folgendes angenommen:

Hypothese 1: Im Vergleich zur Kontrollgruppe zeigt sich bei den Teilnehmenden der Interventionsgruppe nach Durchführung der TGT-Intervention eine signifikante Steigerung (a) des positiven Affekts, (b) des Glücksempfindens sowie (c) eine signifikante Reduzierung des negativen Affekts. 


\subsection{Positive Interventionen und organisationale Ergebnisgrößen}

Arbeitszufriedenheit und organisationales Commitment sind zwei Ergebnisgrößen, die eine hohe Relevanz für den wirtschaftlichen Kontext aufweisen. So sind Beschäftigte mit hoher Arbeitszufriedenheit weniger geneigt, das Unternehmen zu verlassen, und zeigen tendenziell höheres freiwilliges Arbeitsengagement (Kaplan et al. 2009; Ramalho Luz et al. 2018). Studien belegen ferner, dass Commitment positiv mit Arbeitsleistung und Arbeitszufriedenheit zusammenhängt (Gulec und Samanci 2018; Jaramillo et al. 2005). In der Forschung hat sich gezeigt, dass ein hohes Wohlbefinden mit einer hohen Arbeitszufriedenheit und hohem Commitment einhergeht (Albrecht 2012; Connolly und Viswesvaran 2000). Aufgrund des Potenzials der TGT-Intervention, Wohlbefinden zu steigern, postulieren wir folgende Hypothese:

Hypothese 2: Im Vergleich zur Kontrollgruppe zeigt sich bei den Teilnehmenden der Interventionsgruppe nach Durchführung der TGT-Intervention eine signifikante Steigerung (a) der Arbeitszufriedenheit und (b) des organisationalen Commitments.

\subsection{Ressourcen steigern: Positive Interventionen und das Psychologische Kapital}

Aufbauend auf der BBT kann ein positiver Effekt der TGT-Intervention auf arbeitsbezogene Ergebnisgrößen durch den Zuwachs an persönlichen Ressourcen erklärt werden: Durch den Zuwachs an Ressourcen können Beschäftigte z. B. bestimmte Anforderungen besser erfüllen und zeigen höhere Motivation (Hobfoll 2002). Folglich lässt sich annehmen, dass persönliche Ressourcen eine zentrale Rolle einnehmen, um den Wirkungsmechanismus Positiver Interventionen im Arbeitskontext zu erklären. Eine wichtige persönliche Ressource stellt das Psychologische Kapital dar, welches als ein positiver psychologischer Entwicklungszustand, bestehend aus den vier Komponenten Selbstwirksamkeit, Optimismus, Hoffnung und Resilienz, definiert wird (siehe Luthans et al. 2007).

Laut der Metaanalyse von Avey et al. (2011) hängt das Psychologische Kapital positiv mit Wohlbefinden, Commitment, Arbeitszufriedenheit und Arbeitsleistung sowie negativ mit unerwünschten Mitarbeitereinstellungen wie Fluktuationsabsicht zusammen. Bolier et al. (2013) zeigten in ihrer Metaanalyse, dass Interventionen aus der Positiven Psychologie persönliche Ressourcen steigern können. Das Entwicklungspotenzial durch Interventionen konnte auch für das Psychologische Kapital nachgewiesen werden (z. B. Luthans et al. 2008). Insgesamt kann hieraus die Entwicklungsfähigkeit des Psychologischen Kapitals durch das Erleben positiver Emotionen abgeleitet werden, weshalb Folgendes postuliert wird:

Hypothese 3: Im Vergleich zur Kontrollgruppe weisen Teilnehmende der Interventionsgruppe nach Durchführung der TGT-Intervention signifikant höhere Ausprägungen in ihrem Psychologischen Kapital auf. 
Hypothese 4: Die Wirkung der TGT-Intervention auf Arbeitszufriedenheit und organisationales Commitment wird durch den Zuwachs an Psychologischem Kapital erklärt.

\section{Methode}

\subsection{Stichprobe und Studiendesign}

Zur Untersuchung der Hypothesen wurde eine placebo-kontrollierte Studie mit 108 Teilnehmenden $\left(79,6 \%\right.$ weiblich; $\left.\mathrm{M}_{\text {Alter }}=35,55, \mathrm{SD}_{\text {Alter }}=11,01\right)$ an drei Messzeitpunkten durchgeführt. Zunächst beantworteten die Teilnehmenden den ersten Online-Fragebogen und wurden einer von zwei Übungen (TGT-Intervention: $N=63$ oder Placebo-Übung: $N=45$ ) zufällig zugeteilt. Darauf folgte ein siebentägiger Durchführungszeitraum für die jeweilige Übung und im Anschluss eine Post-Befragung. Nach einer Woche folgte der Follow-Up Fragebogen.

Im Rahmen der TGT-Intervention wurden die Teilnehmenden angeleitet, an sieben Tagen jeden Abend für ca. 5-10 Minuten über drei positive Ereignisse des Tages zu berichten und zu begründen, wieso sich die Dinge ereigneten (vgl. Seligman et al. 2005). Um eine Vergleichbarkeit mit anderen Studien zu schaffen, orientierten sich die Instruktion und die Beispiele der Übung an Bono et al. (2013). In der Kontrollbedingung wurden die Teilnehmenden nach Seligman et al. (2005) instruiert, an sieben Tagen jeden Abend für ca. 5-10 Minuten über möglichst frühe Erinnerungen aus der Kindheit zu reflektieren (siehe Mongrain und Anselmo-Matthews 2012).

\subsection{Messinstrumente}

Positiver Affekt $\left(r_{t l}=0,82, r_{t 2}=0,90, r_{t 3}=0,84\right)$ sowie negativer Affekt $\left(r_{t l}=0,81\right.$, $\left.r_{t 2}=0,85, r_{t 3}=0,84\right)$ wurden mit 12 Items der PANAS Skala von Watson et al. (1988) gemessen.

Glücksempfinden $\left(r_{t l}=0,91, r_{t 2}=0,94, r_{t 3}=0,95\right)$ wurde durch das Authentic Happiness Inventory (AHI) von Seligman et al. (2005) erfasst. Auf Grundlage des Reviews von Proyer et al. (2017) wurde eine gekürzte Version des AHI mit 19 Sets à fünf Aussagen eingesetzt, aus denen die Teilnehmenden je eine Aussage auswählen konnten.

Arbeitszufriedenheit $\left(r_{t 1}=0,86, r_{t 2}=0,89, r_{t 3}=0,89\right)$ wurde anhand von zwei Items aus der deutschen Übersetzung (van Dick et al. 2001) der Job Diagnostic Survey (Hackman und Oldham 1980) gemessen.

Organisationales Commitment $\left(r_{t 1}=0,92, r_{t 2}=0,93, r_{t 3}=0,95\right)$ wurde mit neun Items des Organizational Commitment Questionnaire (Porter und Smith 1970) in deutscher Fassung (Maier und Woscheé 2014) gemessen.

Das Psychologische Kapital $\left(r_{t l}=0,86, r_{t 2}=0,89, r_{t 3}=0,91\right)$ wurde anhand von 12 Items der universalen Compound PsyCap Scale (CPC-12; Lorenz et al. 2016) mit einer sechs-stufigen Skala ( 1 = stimme überhaupt nicht zu; $6=$ stimme völlig zu) erfasst. 


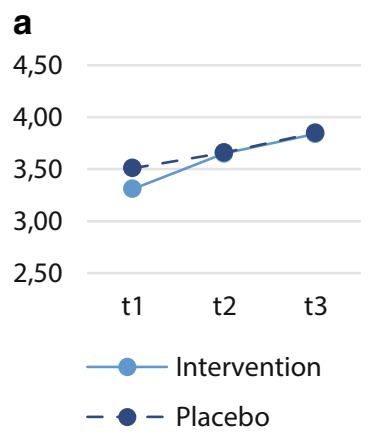

b

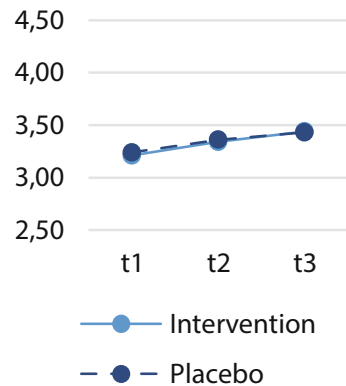

C

3,50

3,00

2,50

2,00

1,50

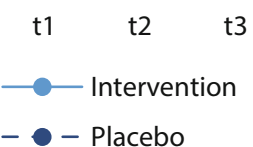

Abb. 1 Mittelwerte des affektiven Erlebens nach Messzeitpunkt und Versuchsbedingung für a Positiver Affekt, b Glücksempfinden, c Negativer Affekt. Anmerkungen. Mittlere Ausprägungen der abhängigen Variablen zu verschiedenen Messzeitpunkten unter Berücksichtigung der Versuchsbedingung; Interventionsgruppe: $N=63$; Kontrollgruppe: $N=45$

Wenn nicht anders berichtet, wurden die Items auf einer Skala von $1=$ stimme überhaupt nicht zu (bzw. trifft gar nicht zu) bis 5=stimme völlig zu (bzw. trifft völlig zu) gemessen.

\subsection{Ergebnisse}

In allen Hypothesen wurde angenommen, dass sich die Veränderung der abhängigen Variablen über die Zeit hinweg signifikant zwischen der Interventions- und der Kontrollgruppe unterscheidet. Zur Überprüfung der Hypothesen wurden Mixed ANOVAs mit zwei Gruppen (Intervention vs. Placebo) als Zwischensubjektfaktor und mit drei Messzeitpunkten als Innersubjektfaktoren (2 Bedingungen * 3 Zeitpunkte) durchgeführt.

\section{Affektives Erleben}

Abb. 1 zeigt die Veränderungen für die beiden Gruppen in ihrem affektiven Erleben. Für positiven Affekt zeigte sich kein signifikanter Interaktionseffekt $(F(2$, 212) $=1,16, p=0,32$, partielles $\left.\eta^{2}=0,01\right)$, jedoch ein signifikanter Haupteffekt für die Zeit $\left(F(2,212)=19,1, p<0,001\right.$, partielles $\left.\eta^{2}=0,15\right)$. Um zu prüfen, zu welchen Zeitpunkten sich der positive Affekt veränderte, wurde der Zeiteffekt in den zwei Gruppen jeweils anhand von paarweisen Vergleichen untersucht. Die Ergebnisse zeigten für die Interventionsgruppe signifikante Unterschiede zwischen den Messzeitpunkten $\mathrm{t} 1$ und $\mathrm{t} 2$ (Differenz $=0,34, p<0,01$ ) sowie $\mathrm{t} 1$ und $\mathrm{t} 3$ (Differenz $=0,53$, $p<0,001)$. In der Kontrollgruppe stieg der positive Affekt lediglich zwischen den Messzeitpunkten $\mathrm{t} 1$ und $\mathrm{t} 3$ signifikant an (Differenz $=0,34 ; p<0,05$ ).

Auch für das Glücksempfinden wurde kein Interaktionseffekt gefunden $(F(1,78$, $188,81)=0,14, p=0,87$, partielles $\eta^{2}<0,01$, korrigiert nach Greenhouse-Geisser), jedoch ein signifikanter Haupteffekt für die Zeit $(F(1,78,188,81)=19,63, p<0,001$, partielles $\eta^{2}=0,16$, korrigiert nach Greenhouse-Geisser). Paarweise Vergleiche des Zeiteffekts in den jeweiligen zwei Gruppen zeigten für die Interventions- 
gruppe signifikante Unterschiede zwischen allen drei Messzeitpunkten (t1 und t2: Differenz $=0,13, p<0,01$; $\mathrm{t} 1$ und $\mathrm{t} 3$ : Differenz $=0,23, p<0,001$; $\mathrm{t} 2$ und $\mathrm{t} 3$ : Differenz $=0,10, p<0,01)$. In der Kontrollgruppe stieg das Glücksempfinden zwischen den Messzeitpunkten $\mathrm{t} 1$ und $\mathrm{t} 3$ signifikant an $($ Differenz $=0,19 ; p<0,05)$.

Für negativen Affekt zeigte sich ein signifikanter Interaktionseffekt zwischen der Gruppenzugehörigkeit und der Zeit $\left(F(2,212)=3,19, p<0,05\right.$, partielles $\left.\eta^{2}=0,03\right)$. Auch hier wurden paarweise Vergleiche eingesetzt, um zu prüfen, zu welchem Zeitpunkt sich der negative Affekt signifikant veränderte. Die Ergebnisse zeigten signifikante Unterschiede zwischen allen drei Messzeitpunkten für die Interventionsgruppe (t1 und t2: Differenz $=-0,41, p<0,01 ; \mathrm{t} 1$ und $\mathrm{t} 3$ : Differenz $=-0,69, p<0,001 ; \mathrm{t} 2$ und t3: Differenz $=-0,28, p<0,05)$. In der Kontrollgruppe zeigten sich zu keinem Zeitpunkt signifikante Veränderungen.

Insgesamt kann nur Hypothese 1c mit einem signifikanten Interaktionseffekt bestätigt werden. Für Hypothese 1a und $1 \mathrm{~b}$ zeigen sich ebenfalls Veränderungen in die postulierte Richtung, jedoch stellenweise sowohl für die Interventions- als auch für die Kontrollgruppe.

\section{Arbeitsbezogene Ergebnisgrößen}

Es zeigten sich keine signifikanten Interaktionseffekte für Arbeitszufriedenheit $\left(F(2,212)=0,61, p=0,55\right.$, partielles $\left.\eta^{2}<0,01\right)$ und organisationales Commitment $\left(F(1,6,169,79)=0,25, p=0,73\right.$, partielles $\eta^{2}<0,01$; korrigiert nach Greenhouse Geisser; Abb. 2). Es wurden ebenfalls keine signifikanten Haupteffekte für die Zeit oder Gruppenzugehörigkeit gefunden. Daher wird Hypothese 2 verworfen.

a

5,00

4,50

4,00

3,50

3,00 b

\section{4,00}

3,50

3,00

2,50

2,00

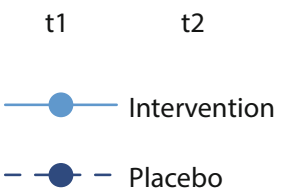

t3

t1

t2

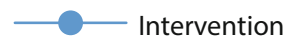

- - Placebo

Abb. 2 Mittelwerte der arbeitsbezogenen Ergebnisgrößen nach Messzeitpunkt und Versuchsbedingung für a Arbeitszufriedenhei, b organisationales Commitment. Anmerkungen. Mittlere Ausprägungen der abhängigen Variablen zu verschiedenen Messzeitpunkten unter Berücksichtigung der Versuchsbedingung; Interventionsgruppe: $N=63$; Kontrollgruppe: $N=46$ 


\begin{abstract}
Abb. 3 Mittelwerte des Psychologischen Kapitals nach Messzeitpunkt und Versuchsbedingung. Anmerkungen. Mittlere Ausprägungen der abhängigen Variable zu verschiedenen Messzeitpunkten unter Berücksichtigung der Versuchsbedingung; Interventionsgruppe: $N=63$; Kontrollgruppe: $N=46$
\end{abstract}

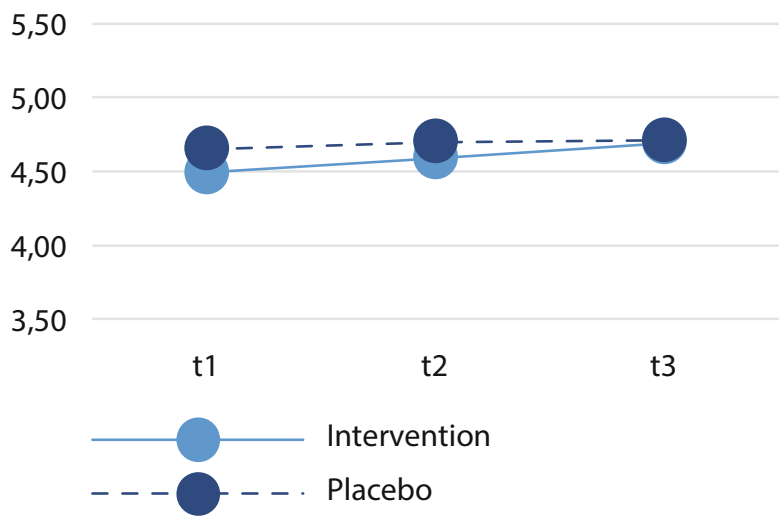

\section{Psychologisches Kapital}

Auch für das Psychologische Kapital konnte kein signifikanter Interaktionseffekt zwischen der Gruppenzugehörigkeit und dem Zeitfaktor gezeigt werden $(F(1,89$, $199,98)=2,13, p=0,12$, partielles $\eta^{2}=0,02$; korrigiert nach Greenhouse-Geisser; siehe Abb. 3). Es wurde jedoch ein signifikanter Haupteffekt der Zeit gefunden $\left(F(1,89,199,98)=7,09, p<0,01\right.$, partielles $\eta^{2}=0,06$; korrigiert nach GreenhouseGeisser). Bei separater Betrachtung weist die Entwicklung des Psychologischen Kapitals in den Gruppen hypothesenkonforme Unterschiede auf: Paarweise Vergleiche in der Interventionsgruppe zeigten signifikante Mittelwertsunterschiede zwischen den Zeitpunkten $\mathrm{t} 1$ und $\mathrm{t} 3$ (Differenz $=0,201, p<0,001$ ) sowie zwischen $\mathrm{t} 2$ und $\mathrm{t} 3$ (Differenz $=0,106 ; p<0,05)$. Für die Kontrollgruppe zeigten sich dagegen keine signifikanten Unterschiede über die Zeit (Abb. 3). Obwohl der Interaktionseffekt für das Psychologische Kapital nicht signifikant wurde und Hypothese 3 nicht angenommen werden kann, veränderte sich das Psychologische Kapital lediglich in der Interventionsbedingung signifikant positiv.

\section{Einfluss des Psychologischen Kapitals auf arbeitsbezogene Ergebnisgrößen}

In Hypothese 4 wurde angenommen, dass die Wirkung der TGT-Intervention auf die arbeitsbezogenen Ergebnisgrößen durch das Psychologische Kapital erklärt wird. Dies sollte mit einer Mediationsanalyse mit dem PROCESS Makro in SPSS geprüft werden. Jedoch zeigten die Ergebnisse der Mixed ANOVA, dass die arbeitsbezogenen Ergebnisgrößen von der TGT-Intervention nicht signifikant beeinflusst wurden. Ebenso zeigte die Mediationsanalyse keinen signifikanten totalen Effekt der Gruppenzugehörigkeit (für Arbeitszufriedenheit: $\beta=-0,01, p=0,96$; für organisationales Commitment: $\beta=0,09, p=0,65)$ wie auch keinen signifikanten indirekten Effekt über das Psychologische Kapital (für Arbeitszufriedenheit: indirekter Effekt = -0,09, $95 \%$-Konfidenzintervall (KI) [0,29; 0,09]; für organisationales Commitment: indirekter Effekt $=-0,08,95 \%$-KI $[-0,28 ; 0,08])$. Dementsprechend muss Hypothese 4 verworfen werden. 


\section{Diskussion und Implikationen für die Praxis}

Im Zentrum des vorliegenden Beitrags steht die Fragestellung, ob und durch welche Mechanismen sich eine Intervention aus der Positiven Psychologie im Arbeitskontext auswirken kann. Im Einklang mit dem aktuellen Forschungsstand (z. B. Wellenzohn et al. 2016) zeigt unsere Studie, dass die TGT-Intervention den negativen Affekt von Mitarbeitenden vermindern kann. Darüber hinaus zeigte sich ebenfalls eine tendenzhafte Verbesserung des positiven Affekts und des Glücksempfindens - jedoch nicht nur in der Interventionsgruppe, sondern, etwas schwächer, ebenfalls in der Kontrollgruppe. Diese Ergebnisse stellen eine Diskrepanz zu vorheriger Forschung dar (z. B. Gander et al. 2013), sind mit Blick auf den kurzen Untersuchungszeitraum allerdings nachvollziehbar (Seear und Vella-Brodrick 2013). Auch in der Studie von Seligman et al. (2005) zur TGT-Intervention verbesserte sich kurzfristig ebenfalls das Wohlbefinden in der Kontrollgruppe, langfristig waren diese positiven Veränderungen jedoch nur in der Interventionsgruppe zu sehen.

Hinsichtlich der Verbesserung von Arbeitszufriedenheit und organisationales Commitment scheint die TGT-Intervention in einem Zeitraum von zwei Wochen nicht wirkungsvoll zu sein. Aufgrund des starken Zusammenhangs zwischen Wohlbefinden und organisationalen Ergebnisgrößen (Kaplan et al. 2009; Lyubomirsky et al. 2005a) scheint es jedoch möglich, dass sich die Effekte der Intervention erst zu einem späteren Zeitpunkt bemerkbar machen (z.B. nach drei Monaten; vgl. Seligman et al. 2005). Zudem deuten die Ergebnisse der paarweisen Vergleiche darauf hin, dass die TGT-Intervention das Potenzial hat, das Psychologische Kapital zu steigern. Dies sollte in zukünftigen Studien validiert werden.

Insbesondere in Verbindung mit bisherigen Forschungsergebnissen und den Annahmen der BBT kann festgehalten werden, dass die TGT-Intervention im Arbeitskontext eingesetzt werden könnte, um das positive affektive Erleben sowie persönliche Ressourcen zu fördern. Positive Auswirkungen eines hohen Psychologischen Kapitals auf organisationale Ergebnisgrößen sind langfristig zu erwarten (vgl. Avey et al. 2011; Fredrickson 1998). Insgesamt ist dabei klar zu empfehlen, die Übung in Organisationen auf freiwilliger Basis über einen längeren Zeitraum fortzuführen, da dies nachweislich die nachhaltigsten Ergebnisse für verschiedenste Ergebnisgrößen aufweist (Gander et al. 2013).

Darüber hinaus verdeutlichen die Ergebnisse, dass mehrere Stellschrauben notwendig sind, um arbeitsbezogene Ergebnisgrößen zu beeinflussen: Nach dem Job Demands-Resources Model spielen neben persönlichen Ressourcen auch arbeitsbezogene Ressourcen und Arbeitsanforderungen eine große Rolle für das Engagement und die Leistung von Beschäftigten (Bakker 2009). Um arbeitsbezogene Ergebnisgrößen möglichst effektiv zu steigern, lässt sich folglich ein ganzheitlicher Ansatz auf Verhaltens- und Verhältnisebene empfehlen, der die persönlichen Ressourcen von Beschäftigten stärkt, aber auch arbeitsbezogene Ressourcen fördert sowie Arbeitsanforderungen angemessen gestaltet (vgl. Lorente et al. 2014). Neben dem Einsatz von Positiven Interventionen sollten Organisationen daher ein unterstützendes Betriebsklima fördern, z.B. indem positive Ereignisse auf der Arbeit, wie positives Feedback, aktiv adressiert und wertgeschätzt werden (Bono et al. 2013). Empfehlenswert ist hier auch, die TGT-Übung zu erweitern und z.B. in Team-Meetings 
$\mathrm{zu}$ integrieren, in denen regelmäßig bewusst über positive Ereignisse und Erfolge berichtet wird. Unterstützungsmechanismen, wie z.B. das Bereitstellen qualitativen Feedbacks oder Coachings durch die Führungskraft, sind weitere Beispiele, um Ressourcen innerhalb des Unternehmens zu fördern (Xanthopoulou et al. 2009). Des Weiteren scheint es effektiver zu sein, verschiedene Positive Interventionen anzubieten und die Beschäftigten die zu ihren Bedürfnissen passende auswählen zu lassen (Parks et al. 2012).

Abschließend lässt sich festhalten, dass der Einsatz von Positiven Interventionen Potenzial zur Förderung des positiven affektiven Erlebens sowie wertvoller persönlicher Ressourcen haben kann. Dies sollte in zukünftigen, längerfristig angelegten Interventionsstudien weiter validiert werden. Insgesamt lässt sich die TGT-Intervention als eine der einfachsten und effektivsten Interventionen aus der Positiven Psychologie (Peters et al. 2013) mit wenig Aufwand gewinnbringend in die Praxis integrieren: Indem Beschäftigte einige Minuten täglich für sich persönlich positive Ereignisse niederschreiben oder mit anderen teilen, können sie neue Denkmuster trainieren und ihren Blick auf das Positive und mögliche Ressourcen in ihrem Umfeld schärfen. So kann sie im Rahmen von Ressourcentrainings oder Coachings eingesetzt werden, um Möglichkeiten herauszuarbeiten, die anhand der Übung identifizierten Ressourcen zu aktivieren und für den Umgang mit Arbeitsanforderungen zu nutzen. Es ist jedoch hervorzuheben, dass die Intervention in Kombination mit anderen, insbesondere mit verhältnisbezogenen Maßnahmen wohl den nachhaltigsten Einfluss auf das Wohlbefinden wie auch auf arbeitsbezogene Ergebnisgrößen hat.

Funding Open Access funding enabled and organized by Projekt DEAL.

Open Access Dieser Artikel wird unter der Creative Commons Namensnennung 4.0 International Lizenz veröffentlicht, welche die Nutzung, Vervielfältigung, Bearbeitung, Verbreitung und Wiedergabe in jeglichem Medium und Format erlaubt, sofern Sie den/die ursprünglichen Autor(en) und die Quelle ordnungsgemäß nennen, einen Link zur Creative Commons Lizenz beifügen und angeben, ob Änderungen vorgenommen wurden.

Die in diesem Artikel enthaltenen Bilder und sonstiges Drittmaterial unterliegen ebenfalls der genannten Creative Commons Lizenz, sofern sich aus der Abbildungslegende nichts anderes ergibt. Sofern das betreffende Material nicht unter der genannten Creative Commons Lizenz steht und die betreffende Handlung nicht nach gesetzlichen Vorschriften erlaubt ist, ist für die oben aufgeführten Weiterverwendungen des Materials die Einwilligung des jeweiligen Rechteinhabers einzuholen.

Weitere Details zur Lizenz entnehmen Sie bitte der Lizenzinformation auf http://creativecommons.org/ licenses/by/4.0/deed.de.

\section{Literatur}

Albrecht, S.L. (2012). The influence of job, team and organizational level resources on employee wellbeing, engagement, commitment and extra-role performance: test of a model. International Journal of Manpower, 33(7), 840-853.

Avey, J. B., Reichard, R. J., Luthans, F., \& Mhatre, K.H. (2011). Meta-analysis of the impact of positive psychological capital on employee attitudes, behaviors, and performance. Human Resource Development Quarterly, 22(2), 127-152. https://doi.org/10.1002/hrdq.20070. 
Bakker, A. B. (2009). Building engagement in the workplace. In C. Cooper \& R. Burke (Hrsg.), The peak performing organization (Bd. 3, S. 50-72). https://doi.org/10.4324/9780203971611.ch3.

Bolier, L., Haverman, M., Westerhof, G. J., Riper, H., Smit, F., \& Bohlmeijer, E. (2013). Positive psychology interventions: a meta-analysis of randomized controlled studies. BMC Public Health, 13(1), 119. https://doi.org/10.1186/1471-2458-13-119.

Bono, J.E., Glomb, T. M., Shen, W., Kim, E., \& Koch, A. J. (2013). Building positive resources: effects of positive events and positive reflection on work stress and health. Academy of Management Journal, 56(6), 1601-1627. https://doi.org/10.5465/amj.2011.0272.

Chakhssi, F., Kraiss, J. T., Sommers-Spijkerman, M., \& Bohlmeijer, E. T. (2018). The effect of positive psychology interventions on well-being and distress in clinical samples with psychiatric or somatic disorders: a systematic review and meta-analysis. BMC Psychiatry, 18(1), 211. https://doi.org/10. 1186/s12888-018-1739-2.

Connolly, J. J., \& Viswesvaran, C. (2000). The role of affectivity in job satisfaction: a meta-analysis. Personality and Individual Differences, 29(2), 265-281. https://doi.org/10.1016/S0191-8869(99)001920 .

Cropanzano, R., \& Wright, T.A. (2001). When a "happy" worker is really a "productive" worker: a review and further refinement of the happy-productive worker thesis. Consulting Psychology Journal: Practice and Research, 53(3), 182-199. https://doi.org/10.1037/1061-4087.53.3.182.

van Dick, R., Schnitger, C., Schartzmann-Buchelt, C., \& Wagner, U. (2001). Der Job Diagnostic Survey im Bildungsbereich. Zeitschrift für Arbeits- und Organisationspsychologie, 45(2), 74-92. https://doi. org/10.1026//0932-4089.45.2.74.

Fredrickson, B.L. (1998). What good are positive emotions? Review of General Psychology, 2(3), 300-319. https://doi.org/10.1037/1089-2680.2.3.300.

Fredrickson, B.L. (2001). The role of positive emotions in positive psychology. The American psychologist, 56, 218-226. https://doi.org/10.1037/0003-066X.56.3.218.

Gander, F., Proyer, R. T., Ruch, W., \& Wyss, T. (2013). Strength-based positive interventions: further evidence for their potential in enhancing well-being and alleviating depression. Journal of Happiness Studies, 14(4), 1241-1259. https://doi.org/10.1007/s10902-012-9380-0.

Gander, F., Proyer, R. T., \& Willibald, R. (2016). Positive psychology interventions adressing engagement, meaning, positive relationship, and accomplishment increase well-being and ameliorate depressive symptoms: a randomized, placebo-controlled online study. Frontiers in Psychology, 7(689), 1-12.

Gulec, D., \& Samanci, S. (2018). The relationship between organisational commitment and job satisfaction in Turkey: a meta-analytical study. International Journal of Human and Behavioral Science, 4(1), 9-21. https://doi.org/10.19148/ijhbs.408904.

Hackman, J. R., \& Oldham, G. R. (1980). Work redesign. Reading: Addison-Wesley.

Hobfoll, S. E. (2002). Social and psychological resources and adaptation. Review of General Psychology, 6(4), 307-324. https://doi.org/10.1037/1089-2680.6.4.307.

Jaramillo, F., Mulki, J.P., \& Marshall, G. W. (2005). A meta-analysis of the relationship between organizational commitment and salesperson job performance: 25 years of research. Journal of Business Research, 58(6), 705-714.

Kaiser, S., Müller-Seitz, G., \& Ringlstetter, M. (2007). Positive Organizational Scholarship. Die Wende der Organisationsforschung zum Guten? zfo Zeitschrift Führung und Organisation, 76, 172-175.

Kaplan, S., Bradley, J.C., Luchman, J. N., \& Haynes, D. (2009). On the role of positive and negative affectivity in job performance: a meta-analytic investigation. Journal of Applied Psychology, 94(1), 162-176. https://doi.org/10.1037/a0013115.

Kaplan, S., Bradley-Geist, J.C., Ahmad, A., Anderson, A., Hargrove, A. K., \& Lindsey, A. (2014). A test of two positive psychology interventions to increase employee well-being. Journal of Business and Psychology, 29(3), 367-380. https://doi.org/10.1007/s10869-013-9319-4.

Lorente, L., Salanova, M., Martínez, I. M., \& Vera, M. (2014). How personal resources predict work engagement and self-rated performance among construction workers: a social cognitive perspective. International Journal of Psychology, 49(3), 200-207. https://doi.org/10.1002/ijop.12049.

Lorenz, T., Beer, C., Pütz, J., \& Heinitz, K. (2016). Measuring psychological capital: construction and validation of the compound Psycap scale (CPC-12). PLOS ONE, 11(4), e152892. https://doi.org/10. 1371/journal.pone.0152892.

Luthans, F., Youssef, C. M., \& Avolio, B. J. (2007). Psychological capital: developing the human competitive edge. Oxford: Oxford University Press.

Luthans, F., Avey, J. B., \& Patera, J.L. (2008). Experimental analysis of a web-based training intervention to develop positive psychological capital. Academy of Management Learning \& Education, 7(2), 209-221. https://doi.org/10.5465/amle.2008.32712618. 
Lyubomirsky, S., King, L., \& Diener, E. (2005a). The benefits of frequent positive affect: does happiness lead to success? Psychological Bulletin, 131(6), 803-855. https://doi.org/10.1037/0033-2909.131.6. 803.

Lyubomirsky, S., Sheldon, K. M., \& Schkade, D. (2005b). Pursuing happiness: the architecture of sustainable change. Review of General Psychology, 9(2), 111-131. https://doi.org/10.1037/1089-2680.9.2. 111.

Maier, G.W., \& Woschée, R. (2014). Deutsche Fassung des Organizational Commitment Questionnaire (OCQ-G). https://doi.org/10.6102/zis10. Zusammenstellung sozialwissenschaftlicher Items und Skalen (ZIS)

Meyers, M.C., van Woerkom, M., \& Bakker, A.B. (2013). The added value of the positive: a literature review of positive psychology interventions in organizations. European Journal of Work and Organizational Psychology, 22(5), 618-632. https://doi.org/10.1080/1359432X.2012.694689.

Mitchell, J., Vella-Brodrick, D., \& Klein, B. (2010). Positive psychology and the Internet: a mental health opportunity. E-Journal of Applied Psychology, 6(2), 30-41. https://doi.org/10.7790/ejap.v6i2.230.

Mongrain, M., \& Anselmo-Matthews, T. (2012). Do positive psychology exercises work? A replication of Seligman et al. (2005). Journal of Clinical Psychology. https://doi.org/10.1002/jclp.21839.

Ouweneel, E., Le Blanc, P. M., \& Schaufeli, W. B. (2013). Do-it-yourself: an online positive psychology intervention to promote positive emotions, self-efficacy, and engagement at work. Career Development International, 18(2), 173-195. https://doi.org/10.1108/CDI-10-2012-0102.

Parks, A.C., Della Porta, M.D., Pierce, R.S., Zilca, R., \& Lyubomirsky, S. (2012). Pursuing happiness in everyday life: the characteristics and behaviors of online happiness seekers. Emotion, 12(6), 1222-1234. https://doi.org/10.1037/a0028587.

Peters, M.L., Meevissen, Y.M.C., \& Hanssen, M. M. (2013). Specificity of the best possible self intervention for increasing optimism: comparison with a gratitude intervention. Terapia Psicológica, 31(1), 93-100. https://doi.org/10.4067/S0718-48082013000100009.

Porter, L. W., \& Smith, F. J. (1970). The etiology of organizational commitment. Irvine: University of California. unpublished paper

Proyer, R. T., Gander, F., Wellenzohn, S., \& Ruch, W. (2017). The authentic happiness inventory revisited: addressing its psychometric properties, validity, and role in intervention studies. Journal of WellBeing Assessment, 1(1-3), 77-96. https://doi.org/10.1007/s41543-018-0006-0.

Ramalho Luz, C.M.D., Luiz de Paula, S., \& de Oliveira, L. M.B. (2018). Organizational commitment, job satisfaction and their possible influences on intent to turnover. Revista de Gestão, 25(1), 84-101. https://doi.org/10.1108/REGE-12-2017-008.

Seear, K.H., \& Vella-Brodrick, D.A. (2013). Efficacy of positive psychology interventions to increase well-being: examining the role of dispositional mindfulness. Social Indicators Research, 114(3), 1125-1141. https://doi.org/10.1007/s11205-012-0193-7.

Seligman, M.E.P. (2010). Flourish: positive psychology and positive interventions. https://tannerlectures. utah.edu/_resources/documents/a-to-z/s/Seligman_10.pdf (Erstellt: 7. Okt. 2010). Zugegriffen: 5. Okt. 2021. The Tanner Lectures on Human Values.

Seligman, M.E.P., Steen, T. A., Park, N., \& Peterson, C. (2005). Positive psychology progress: empirical validation of interventions. American Psychologist, 60(5), 410-421. https://doi.org/10.1037/0003066X.60.5.410.

Seligman, M.E.P., Rashid, T., \& Parks, A.C. (2006). Positive psychotherapy. American Psychologist, 61, 774-788. https://doi.org/10.1037/0003-066X.61.8.774.

Sin, N.L., \& Lyubomirsky, S. (2009). Enhancing well-being and alleviating depressive symptoms with positive psychology interventions: a practice-friendly meta-analysis. Journal of Clinical Psychology, 65(5), 467-487. https://doi.org/10.1002/jclp.20593.

Siu, O.L., Cheung, F., \& Lui, S. (2015). Linking positive emotions to work well-being and turnover intention among Hong Kong police officers: the role of psychological capital. Journal of Happiness Studies, 16(2), 367-380. https://doi.org/10.1007/s10902-014-9513-8.

Tetrick, L. E., \& Winslow, C. J. (2015). Workplace stress management interventions and health promotion. Annual Review of Organizational Psychology and Organizational Behavior, 2(1), 583-603. https:// doi.org/10.1146/annurev-orgpsych-032414-111341.

Tomoff, M. (2017). Positive Psychologie - Erfolgsgarant oder Schönmalerei? Kritisch hinterfragt. Berlin, Heidelberg: Springer.

Watson, D., Clark, L. A., \& Tellegen, A. (1988). Development and validation of brief measures of positive and negative affect: the PANAS scales. Journal of Personality and Social Psychology, 54, 1063-1070. 
Wellenzohn, S., Proyer, R.T., \& Ruch, W. (2016). How do positive psychology interventions work? A short-term placebo-controlled humor-based study on the role of the time focus. Personality and Individual Differences, 96, 1-6. https://doi.org/10.1016/j.paid.2016.02.056.

White, C.A., Uttl, B., \& Holder, M.D. (2019). Meta-analyses of positive psychology interventions: the effects are much smaller than previously reported. PloS one, 14(5), e216588. https://doi.org/10.1371/ journal.pone.0216588.

Wright, T.A., \& Bonett, D. G. (2007). Job satisfaction and psychological well-being as nonadditive predictors of workplace turnover. Journal of Management, 33(2), 141-160. https://doi.org/10.1177/ 0149206306297582.

Xanthopoulou, D., Bakker, A. B., Demerouti, E., \& Schaufeli, W. B. (2009). Work engagement and financial returns: a diary study on the role of job and personal resources. Journal of Occupational and Organizational Psychology, 82(1), 183-200. https://doi.org/10.1348/096317908X285633.

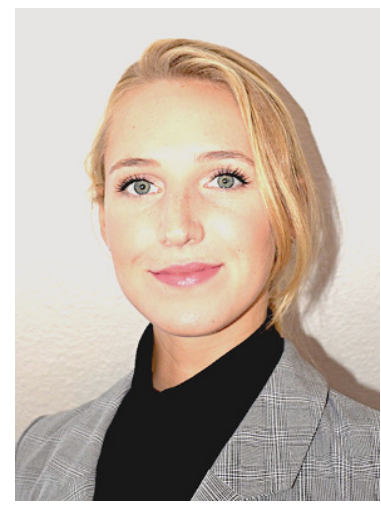

Carmen Elisabeth Lobbe arbeitet in der Wirtschaft als Produktentwicklerin und -beraterin psychologischer Messinstrumente für die Bereiche Personalauswahl, -entwicklung und Wohlbefinden. In ihrem Masterstudium der Wirtschaftspsychologie an der Universität Kassel hat sie ihre Leidenschaft für die Anwendung Positiver Psychologie in der Arbeitswelt entdeckt.

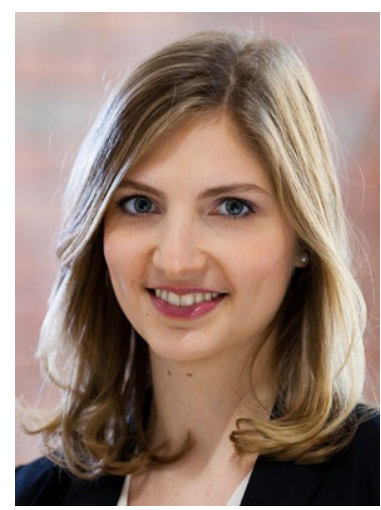

Dr. Kathrin Reinke forscht und lehrt zu den Themen mobiles Arbeiten, Arbeitsstress und Gesundheitsförderung an der TU Darmstadt und der Universität Kassel. Ihr besonderes Interesse liegt im Praxisaustausch und -transfer: Am ArsVitae-Institut Darmstadt bietet sie Beratung und Workshops zu diesen Themen für Unternehmen und Interessierte an. 